\title{
Swept Langmuir probe investigation of a time varying DC discharge
}

\author{
A. Qayyum ${ }^{1}$ (D) M. Usman Naseer ${ }^{1} \cdot$ F. Deeba ${ }^{1}$-S. Ahmad ${ }^{1}$ - S. I. W. Shah ${ }^{1} \cdot$ Mubashair Imran $^{1} \cdot$ S. Hussain ${ }^{1}$. \\ M. Zakaullah ${ }^{2}$
}

Received: 31 July 2020 / Accepted: 21 December 2020 / Published online: 11 January 2021

(c) The Author(s) $2021 \quad$ OPEN

\begin{abstract}
The paper reports on the development and application of a swept Langmuir probe to characterize plasma between two disc-like electrodes. A battery was added to a probe circuit to offset against the cathode fall voltage, and to make the sweep voltage effective at the probe tip. This arrangement allowed the collection of the electron and ion parts of the probe current and the subsequent construction of time-resolved current-voltage $I_{p}(V)$ characteristics with a time resolution of about one millisecond. The probe collected electron current in the lower voltage region of the discharge waveform where it surmounted the cathode fall voltage, whereas the ion current was collected continuously due to an accelerating field for the ions. The results highlighted how the cathode fall voltage limits the collection of the electron and ion parts of the probe current and how to handle the problem with a series battery in the probe circuit. In addition to the swept single-probe, a triple-probe was used simultaneously to compare and validate the results.
\end{abstract}

Keywords Swept Langmuir probe $\cdot$ Triple Langmuir probe $\cdot$ DC-electrode discharge $\cdot$ Time-resolved plasma measurements

\section{Introduction}

The knowledge of the plasma parameters is essential to understand the physical and chemical processes contributing to the plasma-assisted surface modifications $[1,2$, 2]. Langmuir probes (LPs) are a versatile diagnostic tool that can be used to obtain plasma parameters in pulsed as well as turbulent plasmas [3-5]. The technique consists of applying a known voltage (V) to a metallic electrode placed in the plasma and measuring the resulting current to it, $\left(I_{p}\right)[6-8]$. The current-voltage characteristic, $I_{p}(V)$, is used to obtain the plasma parameters including plasma potential, electron temperature, and number density from its different regions. The spatial resolution of conventional probe methods is extremely good, but the time resolution is not sufficient to track the fast changes in transient discharges $[4,6]$. Sweeping the voltage on the probe tip and constructing time-resolved probe characteristics may improve the temporal resolution of probe measurements. However, plasma must behave quasi-stably in every probe sweep half-cycle, i.e. up-sweep and down-sweep, otherwise, the accuracy of the measurements may be compromised depending on the deviation from equilibrium.

Advancing probe technology has enabled the use of triple, multi-pin, and triggered probes to obtain instantaneous values of plasma parameters with a time resolution upto microseconds $[9,10]$. The triple Langmuir probe (TLP) $[11,12]$ is the most favourite multi-pin probe version. It shares the benefits of both double as well as single probe configurations and thus performs real-time measurements with better time resolution. Generally, the triple-probe head is comprised of three conducting tips that enable tracing the $I_{p}(V)$ characteristic instantaneously using the measurements of three distinct points. However, it is not possible to place all three probe tips at the same location, and the plasma conditions could vary from tip to tip in

A. Qayyum, abdul.qaui@gmail.com | ${ }^{1}$ National Tokamak Fusion Program, P O Box 3329, Islamabad, Pakistan. ${ }^{2}$ Center for Advanced Studies in Physics, GC University, Lahore 54000, Pakistan. 
strongly inhomogeneous plasmas. In contrast, the singleprobe can determine the complete $I_{p}(V)$ characteristic at a single point and derivation of the electron energy distribution function (EEDF) $[3,8,9]$. Thus the fast-swept singleprobe can extract information on plasma parameters at one spatial location that cannot be made with a tripleprobe[13-15]. The time-resolved $I_{p}(V)$ characteristics can be constructed at different probe sweep times to determine the electron temperature and the electron number density as a function of time. However, the triple-probe technique is still preferred for fast time measurements inhomogeneous and strongly time-varying plasmas.

Several studies on the application of swept Langmuir probes to time-resolved plasma measurements under various discharge conditions have been reported. Rudakov et al. [16] investigated the effect of electron temperature fluctuations on the measurement of slowly swept probes having a sweep frequency much lower than the characteristic frequencies of plasma fluctuations. The results showed that due to the presence of temperature fluctuations with frequencies higher than the swept frequency, the swept probe tends to read higher than the actual time-averaged electron temperature. This occurs where the time-averaged $I_{p}(V)$ characteristic is used for the fit and plasma potential fluctuations does not correlate with the electron temperature fluctuations. Moreover, when the temperature and plasma potential fluctuations correlate, a slowly swept probe may read either higher or lower than the average electron temperature, depending on the relative phase of the temperature and potential fluctuation and their amplitude. Lobbia et al. [17] studied the factors affecting the time resolution of probe measurements such as sheath formation time, particle transit time, sheath capacitance, stray capacitance, and mutual capacitance for the time-resolved operation of a swept Langmuir probe. These factors limit the time resolution of rapidly swept Langmuir probes due to the inability of the plasma to properly respond to rapidly changing probe bias under steady-state conditions. Theiler et al. [12] investigated the problems faced in obtaining reliable probe data due to phase delay errors, ion sheath expansion, and limited bandwidth of probe sheath dynamics. Based on the nature of the problem, simple guarding circuits were proposed to compensate for the stray capacitance to ground and to improve the time resolution of measurements in strongly time-varying plasmas.

The objective of this work was to develop a fast diagnostic tool for time-resolved plasma measurements in rapidly time-varying plasmas. The proposed modifications in the swept probe technique enable speedy and localized plasma measurements with better accuracy compared with other available techniques by making it a valuable diagnostic implement for dc electrode discharges. This technique also put forward a simple driving circuit to overcome the implications of probe application in the presence of cathode fall voltage between two dc electrodes. The motivation behind this work was to develop a swept single-probe system that would help to understand the time evolution of the microwave discharge in the pre-ionization and later current formation stages during experiments on the Glass Spherical Tokamak (GLAST). The triple-probe technique parallel with the single-probe technique was used in the present research to measure plasma parameters and to compare and verify the results. Moreover, based on the probe measurements, the plasma reactor can be optimized in terms of active species generation and plasma reactivity for surface modifications.

The paper is arranged as follows: Sect. 1 introduces the different Langmuir probe techniques used has been for plasma measurements with their merits and demerits. Section 2 explains the probe theory and its formulation to determine plasma parameters under various discharge conditions. In Sect. 3, experimental details of the plasma generation setup are presented, along with the modified probe circuit and its application to time-varying discharge. Section 4 describes the results with interpretation and discussion. Finally, Sect. 5 summarizes the key findings and implications of the experiment.

\section{Probe theory and analysis}

The probe characteristic, $I_{p}(V)$, describes the dependence of the total probe current on the probe potential and can be constructed experimentally by varying the probe potential relative to a reference electrode. The qualitative interpretation of the probe characteristic provides insight into the sheath dynamics with changing bias voltage. At a large negative probe potential relative to plasma potential $\left(\left|V_{B}\right|<<\left|V_{P}\right|\right)$, the entire probe current is due to positive ions. However, with a decrease in the negative bias voltage, the probe current additionally includes the fast electrons that manage to overcome the retarding field. The superposition of electron current produces an exponential increase in the probe current with increasing bias voltage. Positive ions continue to be collected by the probe until the bias voltage reaches the plasma potential, at which point ions start to be repelled by the probe. For $\left|V_{B}\right|>>\left|V_{P}\right|$, all positive ions are repelled, and thus the entire probe current is attributed to the electron collection. For $\left|V_{B}\right|<\left|V_{P}\right|$, the electrons are partially collected by the probe, and the electron current greatly exceeds the ion current due to their low mass.

The experimental plasma conditions such as collisionality, magnetization level, Debye length, the electron mean free path, and probe size significantly affect 
the accuracy of measurements and interpretation of the probe data. Therefore, it seems necessary to collect information on these quantities to make the measurement more reliable. The plasma particles can be considered as unmagnetized if the cyclotron frequency is less than the collision frequency or their Larmor radii are larger than the mean free paths $\left(r_{L}>\lambda_{m f p}\right)[6,12]$.

$\left(r_{L}=\frac{\sqrt{m_{e} k T_{e}}}{e B}\right)>\left(\lambda_{m f p}=\frac{1}{n_{n} \sigma_{e-n}}\right)$

In the Earth's magnetic field of about $65 \mu \mathrm{T}$, the electron behaves as un-magnetized with $r_{\mathrm{L}}($ at $65 \mu \mathrm{T})=1.6 \times$ $10^{8} \mathrm{~m}>>0.00032 \mathrm{~m}=\lambda_{\mathrm{mfp}}$ and the probe data can be analysed by considering the un-magnetized plasma environment. However, plasma magnetization affects the Larmor radius of the electrons more than that of the ions, and consequently the collection of electrons. It also seems necessary to present information on the ionization fraction of the neutral argon atoms using the density of the neutral argon atoms and using the relation $n_{n}=P / k T_{g}$, where $T_{g}$ is the temperature of the neutral gas, and $P$ is the gas pressure. For typical experimental values of $P=50 \mathrm{~Pa}$ and $T_{g} \approx 300 \mathrm{~K}, n_{n}=1.2 \times 10^{22} \mathrm{~m}^{-3}$, $n_{i}=2.5 \times 10^{18} \mathrm{~m}^{-3}$, the ionization fraction $\left(n_{i} / n_{n}\right)$ is approximately $0.02 \%$. For the sheath collisionless regime, the mean free path of the electrons should be larger than the probe radius and the Debye length [14, 18].The plasma characteristics such as Debye length, $\lambda_{D e}=\left(\frac{\varepsilon_{0} k T_{e}}{e^{2} n_{e}}\right)^{1 / 2}=8.1 \mu \mathrm{m}$, plasma frequency $=14.2 \mathrm{GHz}$, cyclotron frequency $=1.8 \mathrm{MHz}$, and probe radius $=0.125 \mathrm{~mm}$ for the electron number density $\left(2.5 \times 10^{18} \mathrm{~m}^{-3}\right)$ and temperature $(3 \mathrm{eV})$ satisfy the experimental conditions for probe measurements.

The probe current $I_{P}$ is considered positive from the probe to the plasma. Moreover, if $V_{B}$ the biasing voltage of the probe tip and $V_{p}$ the plasma potential in the vicinity of the probe tip, the probe current can be expressed as a sum of electron and ion components for biasing voltages:[6, 14]

$I\left(V_{B}\right)=I_{i}\left(V_{B}\right)+I_{e}\left(V_{B}\right)$

where $I_{i}\left(V_{B}\right)=\left\{\begin{array}{c}-l_{i s} \exp \left[\frac{e\left(V_{P}-V_{B}\right)}{k T_{i}}\right], V_{B} \geq V_{P}, \\ -l_{i s}, V_{B}<V_{P}\end{array}\right\}$, where $I_{e}\left(V_{B}\right)=\left\{\begin{array}{c}l_{e s} \exp \left[\frac{-e\left(V_{P}-V_{B}\right)}{k T_{e}}\right], V_{B} \leq V_{P_{r}} \\ l_{e s}, V_{B}>V_{P}\end{array}\right\}$,

The electron and ion saturation currents, $l_{\text {es }}$ and $l_{\text {is }}$ are given by

$$
\begin{gathered}
I_{e s}=e A_{p} n_{e}\left[\frac{k T_{e}}{2 \pi m_{e}}\right]^{\frac{1}{2}}, \\
\text { andl }_{i s}=I_{\text {Bohm }}=\exp \left(-\frac{1}{2}\right) e n_{i} \sqrt{\frac{k T_{e}}{m_{i}}} A_{p}, T_{e} \gg T_{i}
\end{gathered}
$$

The ion current is determined by the electron current when $T_{e}>>T_{i}$. Here $l_{i}$ is the ion current reaching the probe tip, $l_{e i} I_{e s}$ is the ion and electron saturation currents, $k T_{e}$ is the electron temperature, and $n_{e}$ and $n_{i}$ is the electron and ion density respectively. Moreover, the probe collecting area is denoted by $A_{p}$, the electronic charge is denoted by $e$, and the electronic mass is denoted by $m_{e^{\prime}}$ (all SI units). When the probe potential reaches the plasma potential, the retarding field between the plasma and the probe disappears, and the probe collects almost all electrons reaching it. However, in dc electrode discharges, it is difficult to obtain the entire electron component because of the discharge shifts over the probe tip at higher biasing voltage. In such cases, it is convenient to use the exponential part of the ion current involving the contribution of fast electrons reaching the probe to obtain electron temperature. The electron density may be readily derived from the electron saturation region of the probe characteristic, $l_{p}(V)$. The following are well-known formulae based on probe theory [3-5]:

$k T_{e}[e V]=e\left|\frac{d(\ln / e)}{d V}\right|^{-1}$

$n_{e}=\frac{l_{e s}}{e A_{p} \sqrt{\frac{k T_{e}}{2 \pi m_{e}}}}$

Moreover, the ability to respond to a rapidly changing probe bias also depends on probe sheath transit time, capacitance, and impedance of the measuring circuit. These are the key factors that restrict the temporal response of the probe [17-19]. Apart from swept Langmuir probes [20-23] used by many researchers to obtain timeresolved plasma parameters, the triple-probe can readily provide the temporal profiles of electron temperature and electron density from the voltage difference of positively biased and floating probe tips and the double probe current, respectively, using the following formulae [11-13].

$k T_{e}=\frac{e\left[V_{+}-V_{f}\right]}{0.7}$

$n_{e}=\frac{l_{i s}}{\exp \left(-\frac{1}{2}\right) e A_{p} \sqrt{\frac{k T_{e}}{m_{i}}}}$

Here $I_{\text {is }}$ is the ion saturation current of the differentially biased double probe. $V_{+}$and $V_{f}$ is the potentials of 
the positively biased and floating probes, respectively. A detailed description of the working principle and construction of triple-probes can be found elsewhere [9-11].

\section{Experiment}

To test the swept probe system, a low pressure, pulsed discharge in argon was created between two disc-like brass electrodes arranged in a parallel-plate configuration. The electrodes were powered by a Fluke 410B high voltage dc power supply having triangular-shaped ripples of $50 \mathrm{~Hz}$ to produce a time-varying discharge. The diameter of each electrode was $4 \mathrm{~cm}$ and the spacing between them was also $4 \mathrm{~cm}$. A cylindrically shaped vacuum chamber made of Pyrex glass having a $28-\mathrm{cm}$ height and a $16-\mathrm{cm}$ diameter was used to house the dc electrode assembly. A combined Bayard-Alpert and Pirani Gauge FRG 720made by Agilent was used to monitor the working pressure. A rotary vane pump connected through a controlled valve was used to achieve the required vacuum before argon filling. A resistance of $1-k \Omega$ was connected in series with the power supply to limit the discharge current and record its waveform.

Figure 1 shows a schematic diagram of the plasma generation system together with the swept Langmuir probe biasing circuitry. Here we report on a simple and easy-toconstruct swept probe system that consisted of a Tektronix four-channel variable function generator (AFG 3102C) connected to a DC-to-5 MHz high voltage amplifier (Falco, WMA-300). The plasma characteristics depend on variables such as the voltage across the electrodes, their separation, type of gas, and its working pressure. Moreover, the plasma characteristics and the breakdown voltage apart from the above-mentioned variables also depend on the size of the electrodes. The electrode diameter was chosen

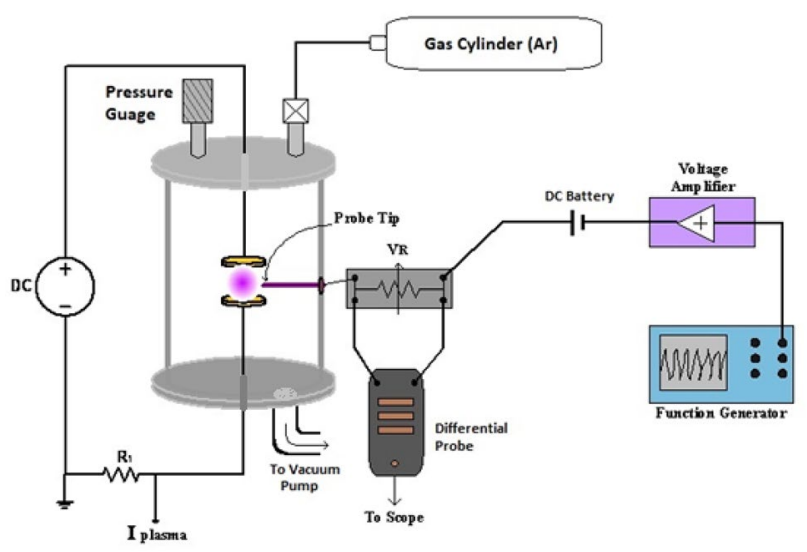

Fig. 1 Schematic diagram of experimental setup for swept probe measurements based on the power supply rating (300 VA), gas type, working pressure, and electrode separation [24].

The triangular-shaped time-varying waveform was chosen to demonstrate the probe's capability for determining the time-resolved current-voltage characteristics and consequently the electron temperature and electron density as a function of time and plasma conditions. A triangularshaped voltage sweep with a frequency of $1 \mathrm{kHz}$ produced by the function generator was fed to the voltage amplifier to amply the probe voltage to the required level. A dc chargeable battery module with alternative polarity was superimposed on the swept voltage to offset the residual cathode fall voltage and thus allow probe measurements. The offset voltage is adjusted to initiate the sweeps from zero volts, in both directions i.e. positive and negative sweeps. The probe current was determined by measuring the voltage across a $10 \mathrm{k} \Omega$ resistor, placed in series with the probe, with differential probe (Rohde\& Schwarz RT-ZD01) coupled with a Rohde and Schwarz 2064 oscilloscope.

The probe was cylindrical and made of tungsten wire. The tip size was chosen to satisfy the criteria for a collisionless, thin sheath regime. The probe tip had a radius of $0.125 \mathrm{~mm}$ and a length of $2 \mathrm{~mm}$. It was shielded by an alumina tube. Because argon was the working gas, the ion bombardment may largely remove the contamination on the probe tips and obviate the need for any special cleaning procedure. The sweep frequency of the function generator was adjusted to $1 \mathrm{kHz}$, which seemed adequate for tracking the temporal variations in the $50 \mathrm{~Hz}$ ripple-dc discharge. The time-resolved probe characteristic, $I_{p}(V)$ was constructed for every half cycle of the voltage sweep, and, accordingly, the electron temperature and density were deduced at different discharge times. The triple-probe technique was simultaneously applied under the same discharge conditions to compare and substantiate the results.

\section{Results and discussion}

Figure 2 shows waveforms of the plasma current, sweep voltage superimposed on the dc battery voltage (with positive polarity), electron part of probe current, sweep voltage superimposed on the dc battery voltage (with negative polarity), and the ion part of probe current. The additional battery allowed the collection of electron probe currents in the region of lower discharge voltage than probe biasing voltage arrived at the ripple ending stage. In the region of higher discharge voltage than the biasing voltage, the collection of electron probe current would not have been possible due to an insufficient accelerating field for electrons. Moreover, with increasing values of probe biasing, the part of the discharge current shifted to the probe instead of the discharge electrode and impose 


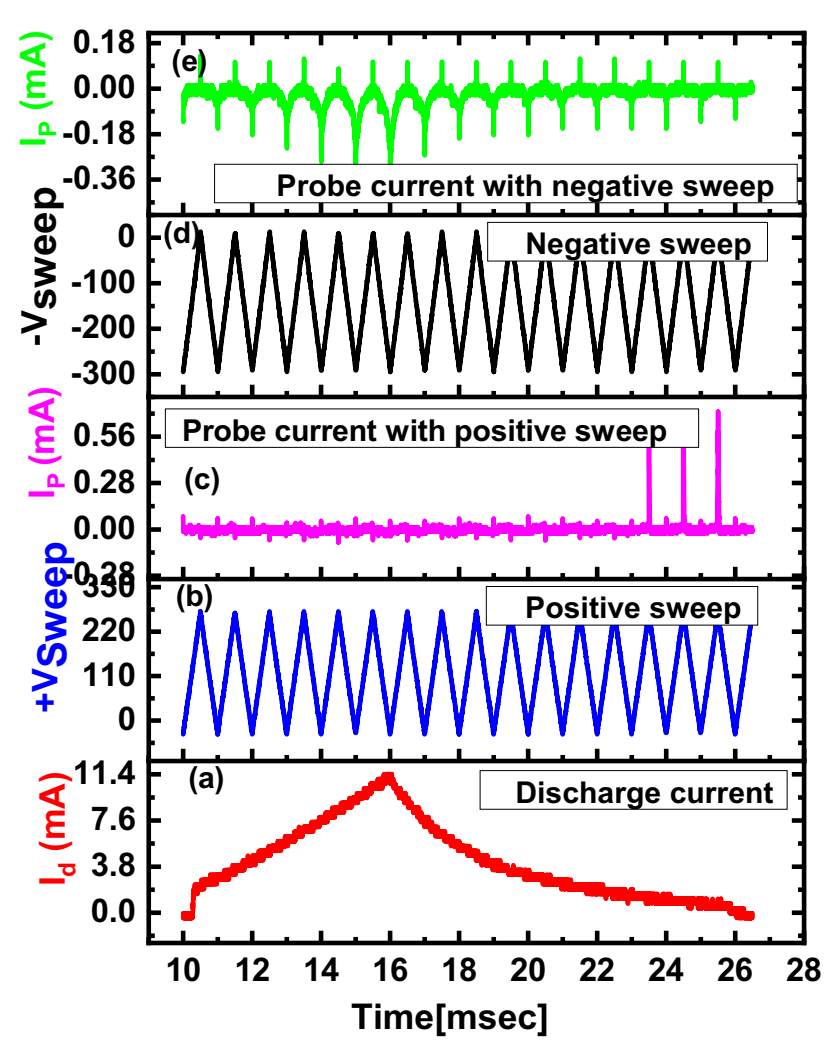

Fig. 2 a Typical plasma current waveform, b voltage sweep with positive offset of battery, c corresponding electron part of probe current, d voltage sweep with negative offset of battery, e corresponding ion part of probe current obtained by sweep frequency of $1 \mathrm{kHz}$ at argon fill pressure of 0.4 mbar, average discharge power of $2.5 \mathrm{~W}$

limits. The ion current was collected continuously for the whole region of the discharge ripple even at a lower bias voltage than the cathode fall voltage.

Figure 3a shows the discharge current and sweep voltage waveforms for argon fill pressure of $0.4 \mathrm{mbar}$, average discharge power of $2.5 \mathrm{~W}$, and sweep frequency of $1 \mathrm{kHz}$. The length of discharge ripple is about sixteen milliseconds superimposed with sixteen sweep cycles with a time resolution of one millisecond. Every half cycle of sweep voltage serves as biasing for the probe to collect the resulting probe current, and construct $I_{p}(V)$ characteristic. Figure $3 b, c, d$ presents the temporal waveform for various discharge voltages with electron parts of probe signals. Results confirm the threshold activation for probe biasing voltage between two dc electrode discharges. The slight difference in the activation of probe biasing for the collection of electron part in the presence of cathode fall may be due to varying local plasma conditions at the probe position. Figure 4 presents $I_{p}(V)$ probe characteristics for two different instantaneous discharge voltages. Electron and ion currents were collected separately, by alternatively sweeping the probe voltage with the battery connected with alternative polarities while the plasma conditions were identical. After acquiring the electron and ion current parts from the individual sweeps, they were combined and the data corresponding to the inactive probe region due to cathode fall voltage (with no probe-current, as shown in Fig. 4) was neglected to construct the complete $I_{P}(V)$ characteristic curve for evaluation of plasma parameters. The resulting plot presents both the electron and ion current parts, for the same plasma conditions. The biasing battery voltages were monitored after each experimental shot and in case of a change in the battery voltage, it was readjusted to the required level before inserting it in the probe circuit.

For cylindrical probes, it is difficult to acquire the typical saturation region at $\left|V_{B}\right|>\left|V_{P}\right|$, and the plasma potential corresponds to the point of deviation from linearity in the electron part of the probe characteristic $[6,18]$. The sheath expansion with increasing bias voltage makes the saturation imperfect because the effective surface area for charged particle collection is the sheath surface area and not the geometric probe area. Thus, with increasing potential on the probes, the minimum impact parameter for particle collection increases and, consequently, more particles are collected. Moreover, at large bias voltage $\left|V_{B}\right|>>\left|V_{P}\right|$, the field turns into an accelerating field, and thus the increase of the electron current with bias voltage violates the rules of conventional probe theory $[14,18]$. The inactive $I_{p}(V)$ probe characteristic region indicates the domain where the collection of probe current is not possible. This happens because in dc glows the plasma potential that is almost equal to cathode fall voltage makes the probe biasing ineffective, and consequently, the probe collects no current.

The electron temperature was determined from the ion part of the $I_{p}(V)$ characteristics $[14,18]$ as a function of time for different argon plasma fill pressure and average discharge power. Figure 5 a shows the ion part of the $I_{p}(V)$ curves for different discharge currents. Here it is clear that slopes of the $I_{p}(V)$ curves depend on the instantaneous values of discharge current and consequently on time. The slope of the $I_{P}(V)$ characteristic has its roots in the energy distribution of the electrons shaping the probe current as a function of biasing voltage. The change in the slope with changing plasma conditions can be credited to the change in EEDF. Therefore, a decrease in the slope of the $I_{P}(V)$ characteristic suggests an increase in the number of energetic electrons in the high energy tail of EEDF that could reach the probe tip even in the presence of a retarding field and constitute a probe current. Figure $5 \mathrm{~b}$ presents the time-variation of $\mathrm{kT}_{\mathrm{e}}$ measured by a swept single Langmuir probe(SLP) and triple Langmuir probe(TLP) for an average discharge power of $2.5 \mathrm{~W}$ and argon fill pressure of 0.4 mbar. The plots show that the time variation of electron temperature closely follows the time variation of 

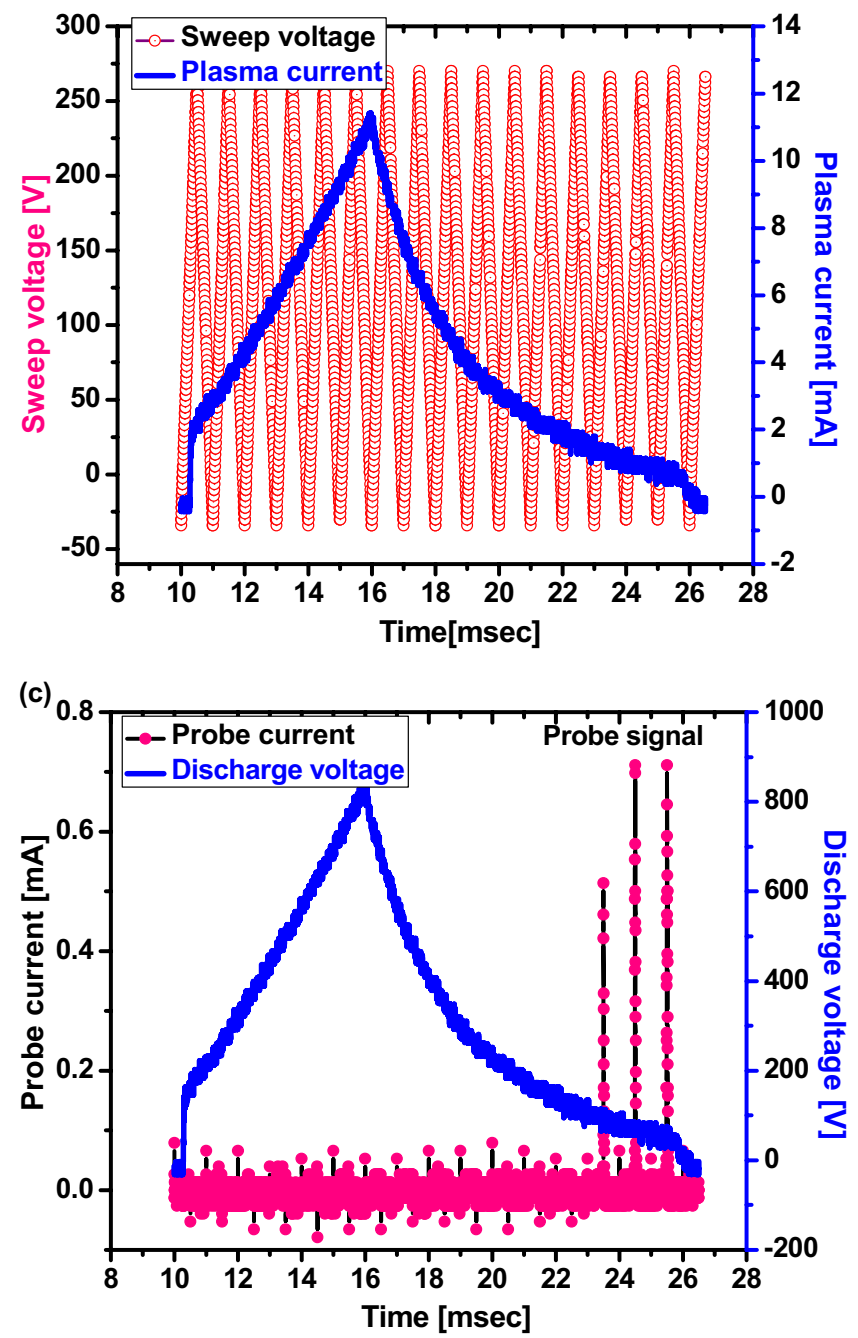

Fig. 3 a Temporal waveforms of plasma current and sweep voltage at argon fill pressure of $0.4 \mathrm{mbar}$, average discharge power of $2.5 \mathrm{~W}$, and sweep frequency of $1 \mathrm{kHz}, \mathbf{b}, \mathbf{c}, \mathbf{d}$ temporal waveform for vari-

discharge current. This is because the energy of electrons contributing to plasma current correlates with the varying current profile. Moreover, the values of electron temperatures obtained by both techniques show almost the same trend. A possible reason for the small discrepancy may be the collection of electrons belonging to a different group of energies. In the case of the triple-probe, the electron current is balanced by the ion current in the double probe and, therefore, it can never exceed the ion current involving only an energetic group of electrons. This behaviour can also be attributed to both the sheath expansion around the probe tip that enlarges the collecting area and the collision process occurring within the probe sheath $[6,25]$.

sInitially, when the probe tip is exposed to the plasma environment, the electrons as more mobile of plasma
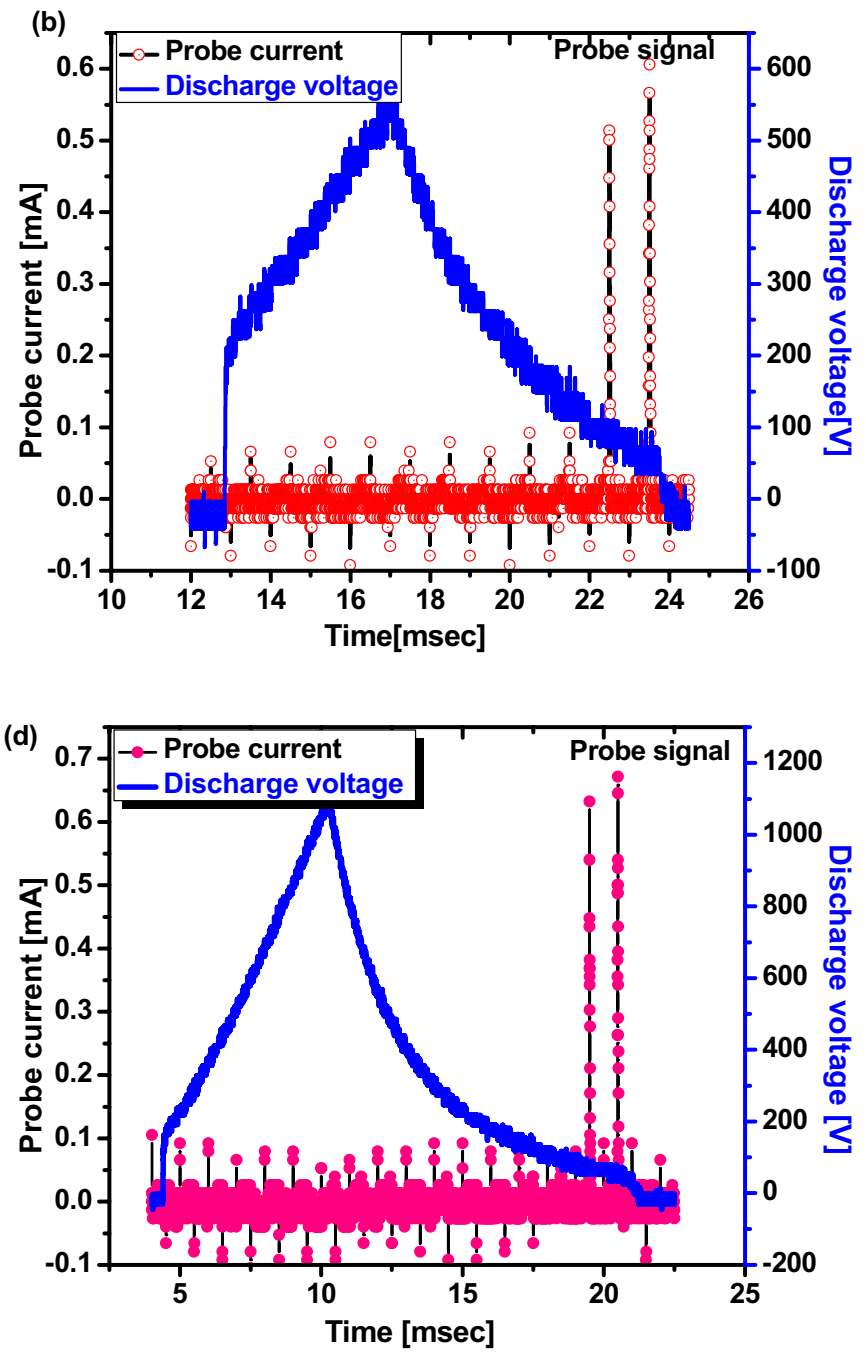

ous discharge voltages with electron part of probe signals showing the activation threshold for probe biasing voltage in presence of resident cathode fall voltage between two disc-like dc electrodes

reach the probe tip first and charge it negatively. This negatively charged probe tip repels further incoming electrons and attracts positive ions, and thus a dynamic equilibrium is established constituting the net current by the electrons and ions equal to zero. As no net current flows to the probe under these conditions, we call it a floating probe, and the corresponding potential is called floating potential. When biasing is applied to the probe tip and increased gradually, the retarding potential $\left(\left|\mathrm{V}_{\mathrm{P}}\right|-\right.$ $\left.\left|V_{B}\right|\right)$ decreases continuously and finally disappears. In the beginning, only the energetic electrons can overcome the potential barrier and reach the probe tip, then slower ones can at lower retarding potential and higher probe biasing. Thus, the shape of the retarding region of $I_{p}(V)$ characteristic resulting from the probe current as a 

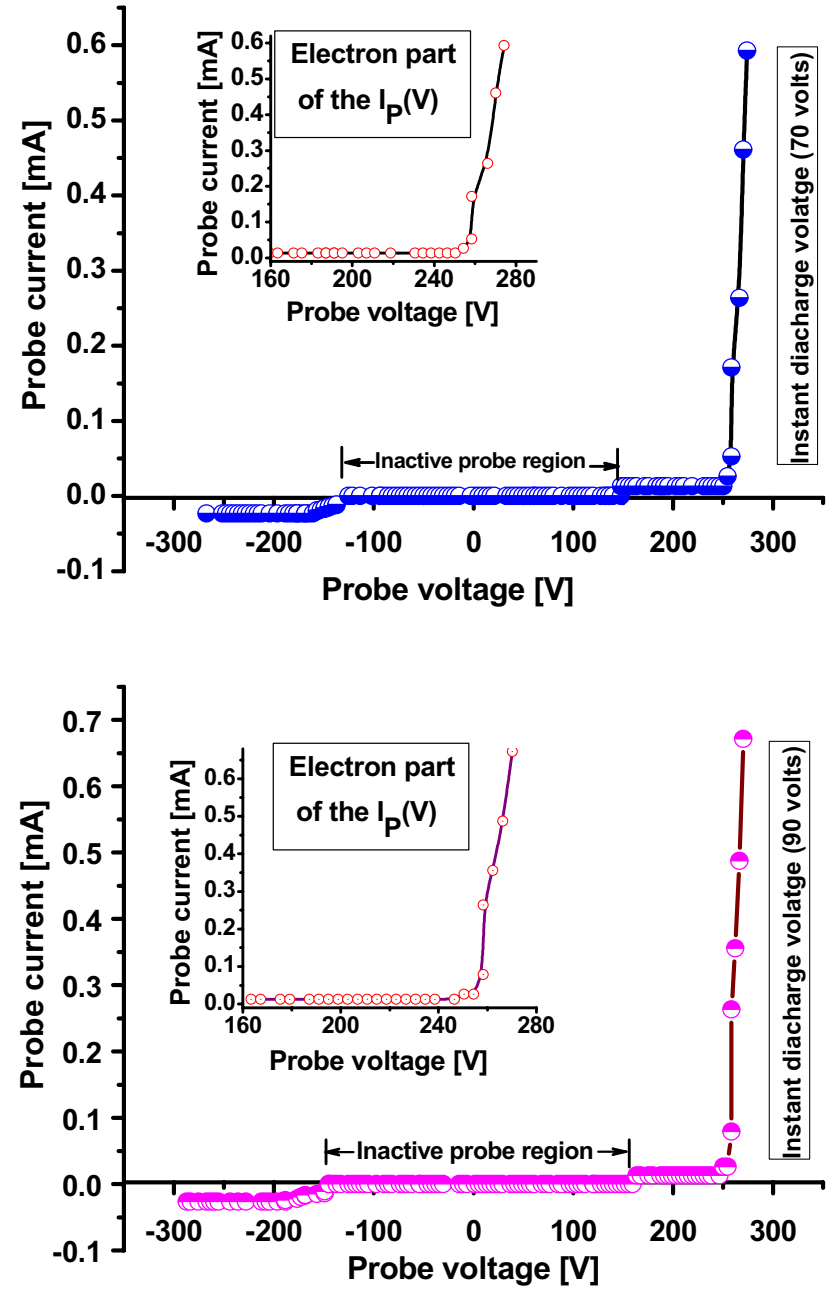

Fig. $4 \mathrm{I}(\mathrm{V})$ probe characteristics for two different values of instantaneous discharge voltages, constructed by combining the ion and electron parts of the probe current collected with alternative polarities to provide offset against cathode fall voltage, and to collect electron current and ion current successively at argon fill pressure of $0.4 \mathrm{mbar}$ (The figure insets describe the electron part of the I(V) characteristic)

function of biasing voltage offers an effective representation of electron energy distribution [14-16].

Figure 6a shows the $I_{p}(V)$ characteristics recorded at peak discharge values for different argon fill pressures at a fixed average discharge power of $2.5 \mathrm{~W}$. The plots reveal that the number of energetic electrons decreases with increasing argon fill pressure that may be attributed to the effective collisional transfer of energy from electrons to other plasma species. Thus, the energetic electrons are preferentially cooled and not able to reach the probe tip by overcoming the retarding field. This shows the reduction in electron temperature with increasing argon fill pressure. The $I_{P}(V)$ plots in Fig. $6 \mathrm{~b}$ reveal the effect of discharge power on their slopes and, consequently, to the electron's energy distribution. Plots show that average

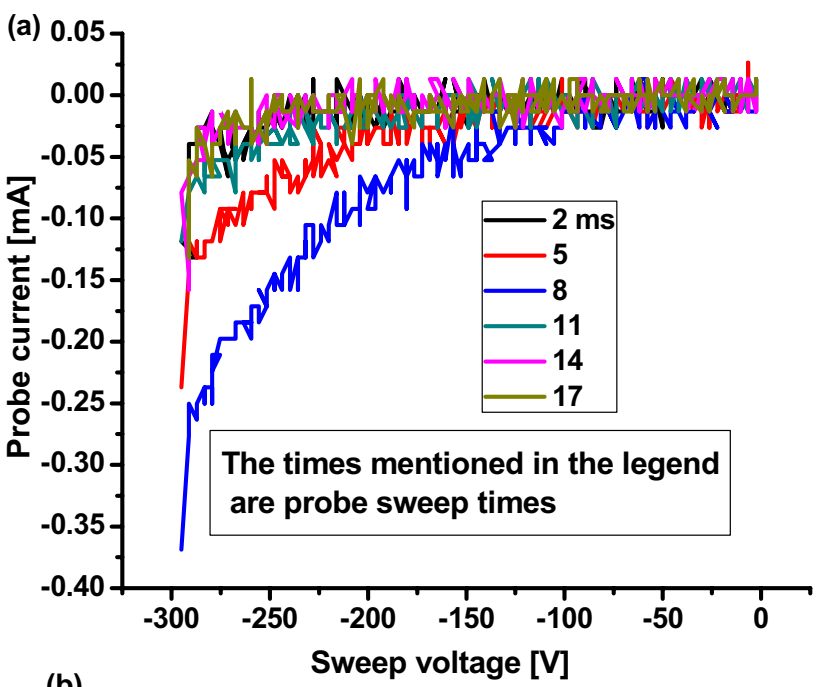

(b)

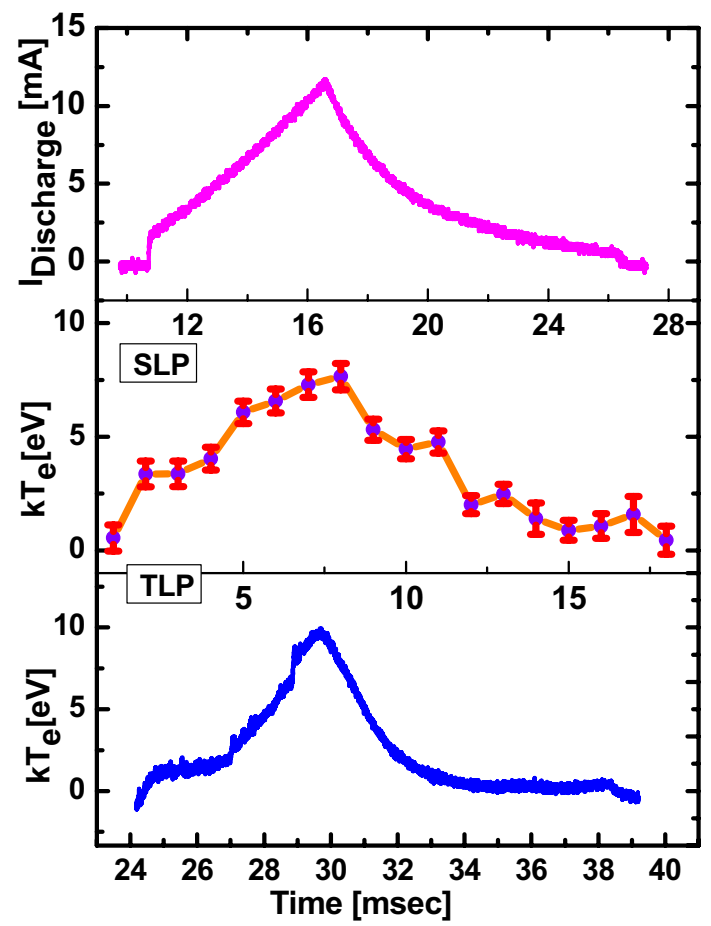

Fig. 5 a Time-variation of $I(V)$ curves recorded at different probe sweep times at different values of plasma current, $\mathbf{b}$ time-variation of $\mathrm{kT}_{\mathrm{e}}$ measured by SLP and TLP at average discharge power of $2.5 \mathrm{~W}$ and argon fill pressure of $0.4 \mathrm{mbar}$

discharge power influences the overall bulk electron's energy contrary to the case of changing fill pressure where high energy electrons are influenced preferentially. The effective ionization process may raise the electron number accessible to reach the probe tip at the same probe biasing.

Figure 7a shows the dependence of electron temperature and number density on argon fill pressure at a fixed average discharge power. The electron temperature 

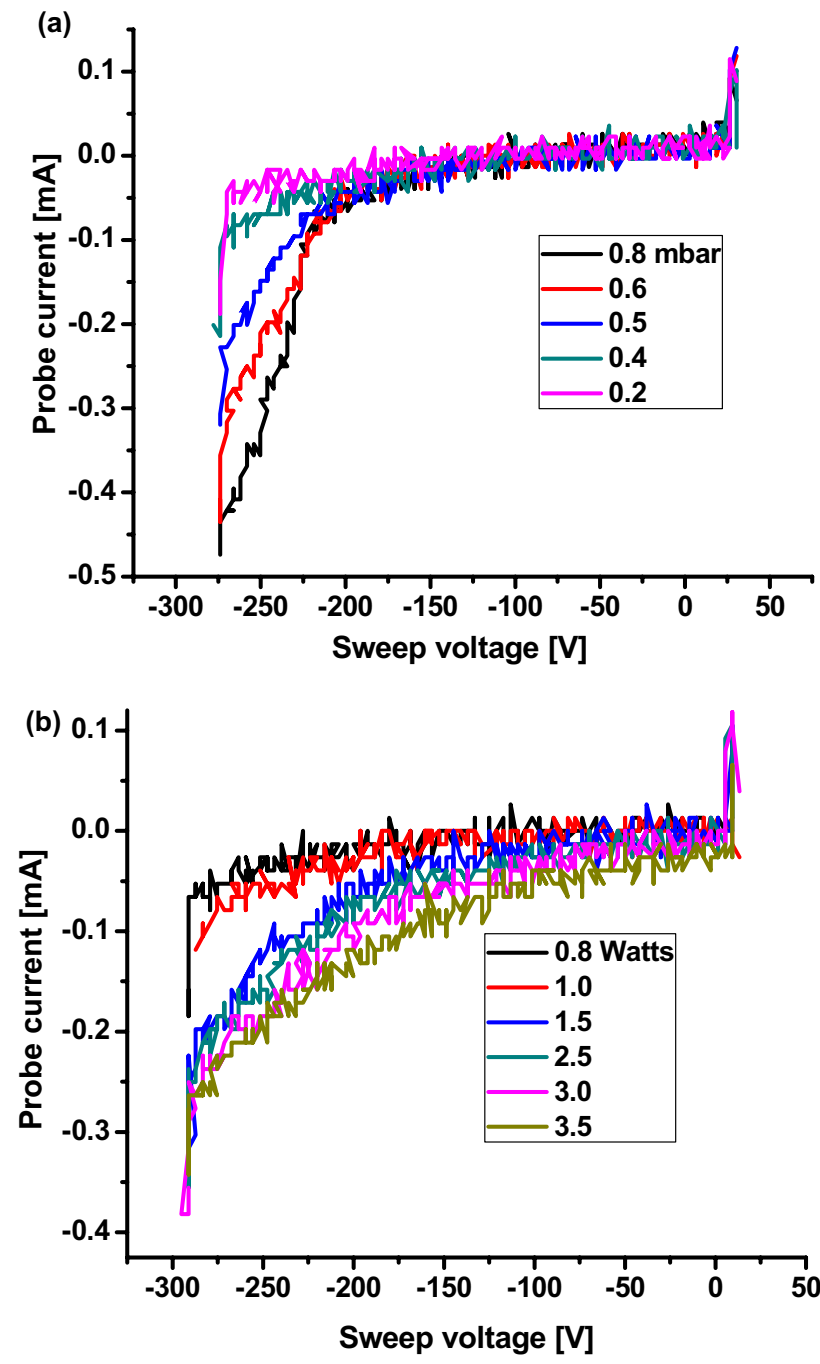

Fig. 6 a I(V) curves for various argon pressures (0.2-0.8 mbar) at average discharge power of $2.5 \mathrm{~W}, \mathbf{b} \mathrm{I}(\mathrm{V})$ curves for various average discharge powers at argon fill pressure of $0.4 \mathrm{mbar}$

decreased(from 3.21 to $2.12 \mathrm{eV}$ ) with discharge pressure (0.2-0.8 mbar).This is because of the collisional transfer of energy from electrons at a higher pressure and consequent cooling of electrons. The electron density increased(from $0.82 \times 10^{12}$ to $2.54 \times 10^{12} \mathrm{~cm}^{-3}$ ) with discharge pressure (0.2-0.8 mbar). This might be due to enhanced electronimpact ionization at a given discharge pressure. Figure $7 \mathrm{~b}$ presents the variation of electron temperature and number density with average discharge power determined at the peak value of the triangular current waveform. The electron temperature increased from 1.82 to $4.74 \mathrm{eV}$ with discharge power (0.7-3.5 W), whereas electron number density increased from $0.82 \times 10^{12}$ to $2.75 \times 10^{12} \mathrm{~cm}^{-3}$ with discharge power (0.7-3.5 W),respectively. This shows an increase in electron energy due to higher electric fields energizing electrons and producing new electrons due to
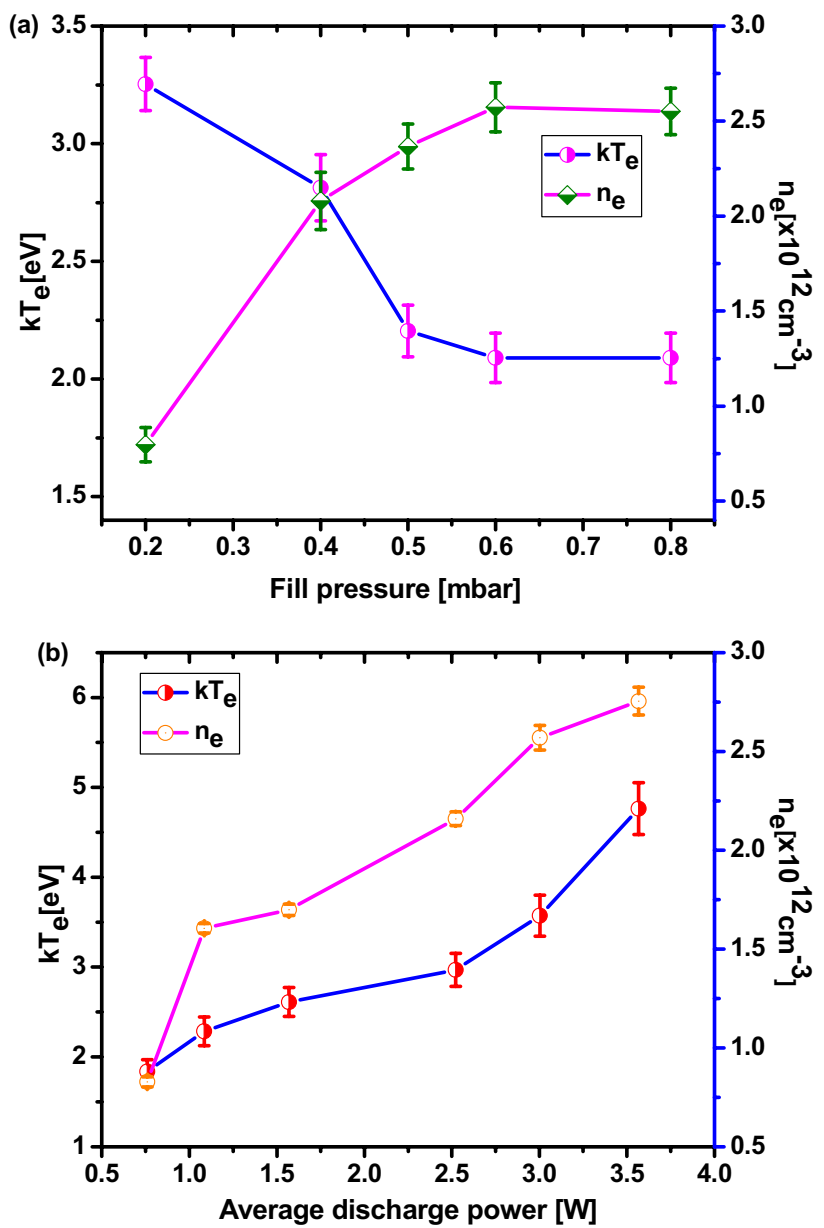

Fig. 7 aVariation of average $K T_{e}$ and $n_{e}$ with various gas fill pressures (0.2-0.8 mbar) at average discharge power of $2.5 \mathrm{~W}$, b Variation of average $\mathrm{KT}_{\mathrm{e}}$ and $\mathrm{n}_{\mathrm{e}}$ with various average discharge powers at argon fill pressure of $0.4 \mathrm{mbar}$

electron-impact ionization. The electrons receive energy from the electric fields and make inelastic collisions producing electron-ion pairs.

The electron density shows a relatively strong dependence on the discharge power with an increase of 235 percent in comparison with electron temperature, which increases 160 percent with the discharge power. A similar dependence of electron temperature and electron density on RF discharge power and discharge pressure has been reported by Zhao et al. [26]. As the discharge pressure increased, the electron collision frequency $v_{e e}$ increased resulting in higher electron density and lower electron temperature due to $\vartheta_{e e} \propto n_{e} T_{e}^{-3 / 2}$. Godyak et al. [7] reported the effect of discharge pressure on effective electron temperature and electron number density measured from the EEDF using the single-probe technique in argon. The plasma density was found to increase with discharge pressure whereas electron temperature was found to decrease. They also proposed that at higher electron 
density, there exists a larger energy range where the EEDF coincides with the Maxwellian distribution and better matched the probe theory assumption. Electron-electron collisions provide a strong Maxwellizing effect. Moreover, in low-pressure plasmas, the probe sheath can be safely assumed to be collisionless for electron dynamics, and the overlap of plasma sheaths forming around the adjacent probes is negligible [27].

Mainly two factors could affect the accuracy of the instant probe measurements. Firstly, any variation in the local plasma conditions (potential, density, and temperature) during the sweep cycle induces errors in the plotting of the $I_{P}(V)$ characteristics and consequently in the plasma parameters. It is not possible practically to keep plasma conditions constant during the probe sweep time. Therefore, a hysteretic effect occurs during the upsweep and downsweep probe measurement because of the timedependent variations in plasma properties related to the $I_{P}(V)$ characteristic. Hence, it is only possible to get averaged values over the upsweep or downsweep half-sweep cycle, rather than instantaneous values. The accuracy of the temporal measurements can be improved significantly by increasing the sweep frequency and data sampling rate using appropriate circuitry.

As pointed out earlier, apart from sweep frequency, the time resolution also depends on plasma sheath transit time, sheath capacitance, and stray capacitance of the circuit. Secondly, errors could be introduced due to deviations of the electron distribution from the Maxwellian, because the fitting procedures assume a Maxwellian electron energy distribution function. However, in reality, the EEDF deviates from the Maxwellian. Therefore, the fitting errors of the $I_{p}(V)$ characteristic in the chosen time window could affect the accuracy of the derived parameters. Herein the fitting errors of the experimental $I_{p}(V)$ characteristic curves using the standard exponential curve fit were estimated to be in the range of 4-6 percent. A possible reason for the discrepancy between the single and triple-robe measurements is that in the triple-probe configuration, close to the ion saturation region, the three tips have different ion sheath structures due to different potentials on the probe tips. Thus, the expansion of the ion sheath with bias voltage changes the effective collection surface area and leads to the variation of probe currents.

\section{Conclusion}

The application of a swept Langmuir probe to plasma between two dc electrodes and the collection of probe currents is not straightforward. However, this situation can be handled readily using an additional dc battery in the probe circuit that provides an offset against cathode fall voltage and makes probe measurements possible. The temporal profile of the electron temperature closely followed the discharge current profile. The plasma parameters depended on the shape of the discharge current, the argon fill pressure, and the average discharge power. The experimental findings show the capability of the probe system for time-resolved plasma measurements. This technique is more suitable for slow time-varying plasmas to have fluctuation frequency well below the sweep frequency to provide more reliable values of the plasma parameters.

Acknowledgements Financial support from the Planning Commission of Pakistan and IAEA through Coordinated Research Project (CRP) "Utilization of the Network of Small and Medium Size Magnetic Confinement Fusion Devices for Fusion Research (F13019)" under Contract No.22771 is acknowledged. The authors thank Mr. Liaqat Ali DG TNO for their continuous support and encouragement.

\section{Compliance with ethical standards}

Conflict of interest The authors declare that they have no known competing financial interests or personal relationships that could have appeared to influence the work reported in this paper.

Open Access This article is licensed under a Creative Commons Attribution 4.0 International License, which permits use, sharing, adaptation, distribution and reproduction in any medium or format, as long as you give appropriate credit to the original author(s) and the source, provide a link to the Creative Commons licence, and indicate if changes were made. The images or other third party material in this article are included in the article's Creative Commons licence, unless indicated otherwise in a credit line to the material. If material is not included in the article's Creative Commons licence and your intended use is not permitted by statutory regulation or exceeds the permitted use, you will need to obtain permission directly from the copyright holder. To view a copy of this licence, visit http://creativecommons .org/licenses/by/4.0/.

\section{References}

1. Bilal M, Qayyum A, Ahmad Z, Ahmad K, Naseer MU, Shah A, Mahmood A, Rehman MM, Hussain S (2020) Active screen plasma nitriding of Al-Si eutectic alloy and evaluation of compound coatings. Appl Phys A 126:159

2. Deeba F, Qayyum A, Naseer MU, Hussain S (2020) Optical actinometric measurements of nitrogen impurity in $\mathrm{Ar} / \mathrm{He}$ microwave discharge during wall cleaning of MT-I spherical tokamak. Vacuum 182:109672

3. Krishna M, Kumar SM (2019) Synthesis of nanocrystalline titanium nitride by microwave plasma technique. SN Appl Sci 1:833

4. Chen FF (2003) Lecture notes on langmuir probe diagnostics, mini-course on plasma diagnostics. IEEE-ICOPS meeting, Jeju, Korea

5. Wang Y, Zhao W, Zhao L, Nie L, Zhong G, Watts C, Gunn JP (2020) Design of electronics system for Langmuir probes on ITERFusion. Eng Des 152:111429 
6. Mirzaei HR, Amrollahi R, Ghasemi M (2020) Optimizing the plasma startup through ECR plasma pre-ionization in Taban Tokamak via triple Langmuir probe. Fusion Eng Des 150:111362

7. Godyak VA, Alexandrovich BM (2015) Comparative analyses of plasma probe diagnostics techniques. J Appl Phys 118:233302

8. Godyak VA, Demidov VI (2011) Probe measurements of electronenergy distributions in plasmas: what can we measure and how can we achieve reliable results? J Phys D Appl Phys 44:233001

9. Popov TK, Dimitrova M, Ivanova P, Kovačič J, Gyergyek T, Dejarnac R, Stöckel J, Pedrosa MA, López-Bruna D, Hidalgo C (2016) Advances in Langmuir probe diagnostics of the plasma potential and electron-energy distribution function in magnetized plasma. Plasma Sour Sci Technol 25:033001

10. Qayyum A, Deeba F, Naseer MU, Ahmad S, Javed MA, Hussain S (2018) A photodiode array and Langmuir probe for characterizing plasma in GLAST-III tokamak device. Measurement 125:56

11. Hussain S, Qayyum A, Ahmad Z, Ahmad S, Khan R, Naveed MA, Ali R, Deeba F, Vorobyov GM (2017) Electrical and optical measurements in the early hydrogen discharge of GLAST-III. Plasma Sci Technol 19:085103

12. Qayyum A, Ahmad N, Ahmad S, Deeba F, Ali R, Hussain S (2013) Time-resolved measurement of plasma parameters by means of triple probe. Rev Sci Instrum 84:123502

13. Theiler C, Furno I, Kuenlin A, Marmillod P, Fasoli A (2011) Practical solutions for reliable triple probe measurements in magnetized plasmas. Rev Sci Instrum 82:013504

14. Chen SL, Sekiguchi T (1965) Instantaneous direct-display system of plasma parameters by means of triple probe. J Appl Phys 36:2363

15. Merlino RL (2007) Understanding Langmuir probe current-voltage characteristics. Am J Phys 75:1078

16. Lobbia RB, Gallimore AD (2010) High-speed dual Langmuir probe. Rev Sci Instrum 81:073503

17. Rudakov DL, Boedo JA, Moyer RA, Stangeby PC, McLean A, Watkins JG (2004) Effect of electron temperature fluctuations on slowly swept Langmuir probe measurements. Rev Sci Instrum 75:4334

18. Lobbia RB, Gallimore AD (2010) Temporal limits of a rapidly swept Langmuir probe. Phys Plasmas 17:073502
19. Kagan YM, Perel VI (1964) Probe methods in plasma research. Sov Phys Usp 81:409

20. Hershkowitz N (2005) Sheaths: more complicated than you think. Phys Plasmas 12:055502

21. Riccardi C, Longoni G, Chiodini G, Fontanesi M (2001) Comparison between fast-sweep Langmuir probe and triple probe for fluctuations measurements. Rev Sci Instrum 72:461

22. Chiodini G, Riccardi C, Fontanesi M (1999) A 400 kHz, fast-sweep Langmuir probe for measuring plasma fluctuations. Rev Sci Instrum 70:2681

23. Enling T, Shenghai $X$, Minghai $Y$, Lexin $L$ (2012) Sweep Langmuir probe and triple probe diagnostics for transient plasma produced by hypervelocity impact. Plasma Sci Technol 14:747

24. Mitov M, Bankova A, Dimitrova M, Ivanova P, Tutulkov K, Djermanova N, Dejarnac R, Stöckel J, Popov TK (2012) Electronic system for Langmuir probe measurements. J Phys Conf Ser 356:012008

25. Held J, Maab PA, der Gathen VS-Von, Keudell von A (2020) Electron density, temperature and the potential structure of spokes in HiPIMS. Plasma Sour Sci Technol 29:025006

26. Estrin FL, Karkari SK, Bradley JW (2017) Triple probe interrogation of spokes in a HiPIMS discharge. J Phys D: Appl Phys 50:295201

27. Zhao P, Li D, Chen D, Liu K, Li X, Wang H, Zuo C, Fan M (2017) Plasma characterization of a test setup of RF-driven negative ion source by a Langmuir probe system. Nucl Instrum Method Phys Res A 858:90

28. Jauberteau JL, Jauberteau I (2008) Determination of the electron density in plasma by means of a floating double probe. Rev Sci Instrum 79:033505

Publisher's Note Springer Nature remains neutral with regard to jurisdictional claims in published maps and institutional affiliations. 